\title{
Evaluation of Hepatitis B Vaccine
}

\author{
S. KRUGMAN ${ }^{1}$
}

The development of rapid methods to identify the etiology of various infectious diseases is an important objective. However, in the development and evaluation of vaccines, caution and prudence rather than rapidity are essential. The acceleration of vaccine development by so-called "crash programs" can prove to be disastrous.

The history of the development of polio, measles, and rubella vaccines reveals that the time interval between identification and cultivation of the agents and subsequent licensure of the vaccines ranged between 7 and 16 years. Inactivated poliovaccine was licensed for use in 1956 and live attenuated poliovaccine in 1961, 7 and 11 years respectively after successful cultivation of the virus in 1949 . Measles vaccine was licensed in 1963, 9 years after the growth of the virus in cell culture. The time interval between propagation of rubella virus in 1962 and subsequent licensure of the attenuated vaccine in 1969 was 7 years. The development of these viral vaccines required the successful propagation of large quantities of virus for inactivation or attenuation.

Unlike polio, measles, and rubella viruses, the successful cultivation of hepatitis B virus (HBV) has not been achieved. Nevertheless, it has been possible to develop, evaluate, and license various hepatitis B vaccines. The sequence of events that culminated in this important development occurred over a span of 16 years. The many milestones that led to this achievement stemmed from the contributions of various investigators during the period 1965-1981.

\section{History of the Development of the Hepatitis B Vaccine}

The first and most important milestone was the discovery of Australia antigen by Blumberg et al. (1965). The antigen that they detected in the blood of an Australian aborigine subsequently proved to be associated with hepatitis B infection (Prince 1968). In 1970, Dane et al. (1970) identified 42-nm, double-shelled particles in the blood of patients with Australia antigen-associated hepatitis. They detected these particles by immune electronmicroscopy. Later, it became apparent that the so-called Dane particle was HBV. It also became apparent that Australia antigen was immunologically identical with the envelope or surface component of $\mathrm{HBV}$ and immunologically distinct from the core component of HBV.

1 New York University Medical Center, 550 First Avenue, New York, NY 10016, USA 


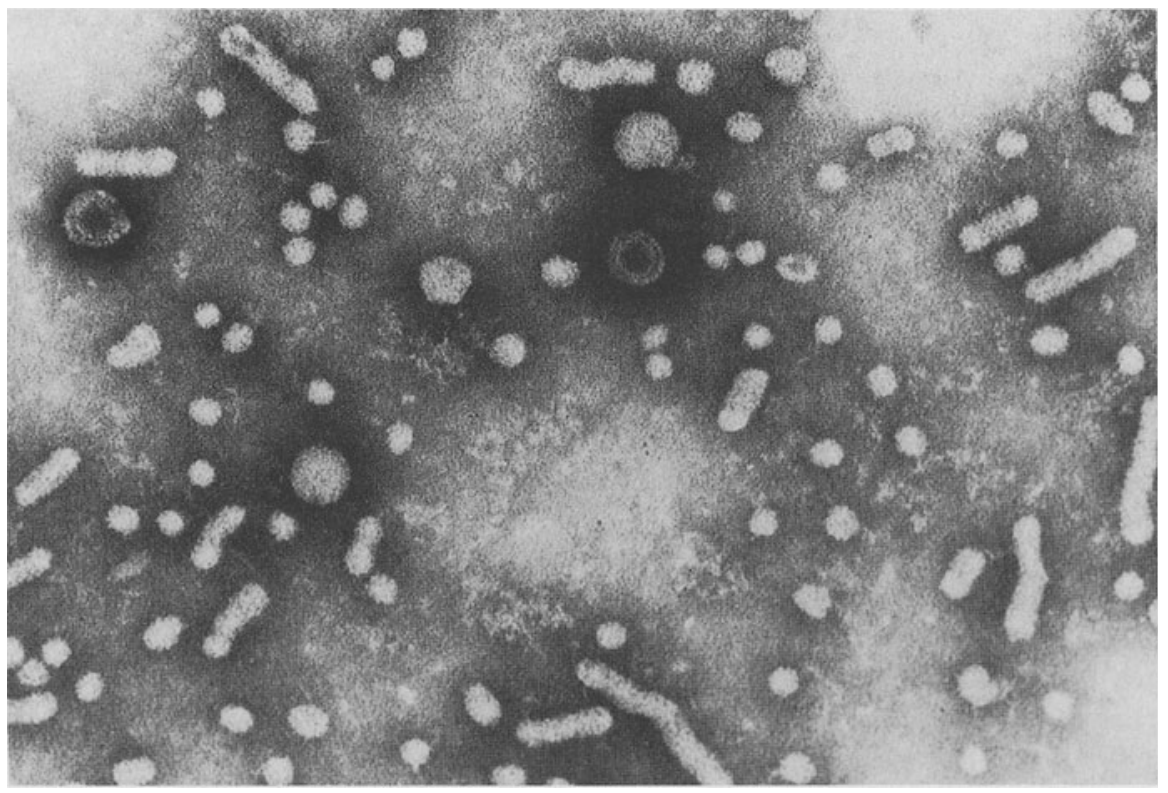

Fig. 1. Electronmicrograph of serum containing HBV particles, $42 \mathrm{~nm}$ in diameter, and HBsAg particles that are either 20-nm spheres or tubular structures of similar diameter and variable length

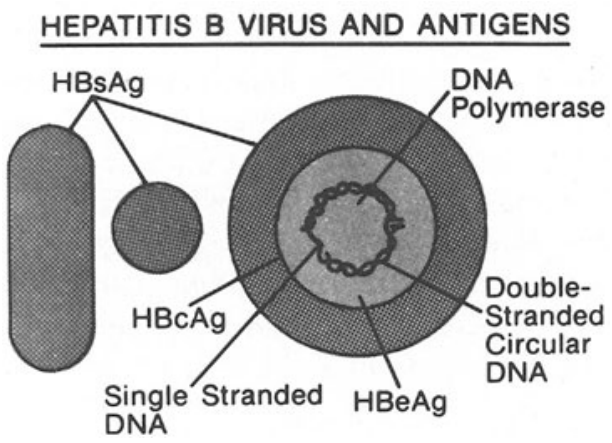

Fig. 2. Schematic illustration of hepatitis $B$ virus (HBV) and its antigens, $\mathrm{HBsAg}, \mathrm{HBcAg}$, and $\mathrm{HBeAg}$

An electronmicrograph of $\mathrm{HBV}$ infected blood reveals three morphologically distinct particles: 20 -nm spheres, tubular structures of similar diameter and variable length, and 42-nm HBV (Dane) particles (Fig. 1). The spherical and tubular particles are immunologically identical with the surface component of $\mathrm{HBV}$ and are now designated hepatitis B surface antigen (HBsAg). The core component contains two immunologically distinct antigens, hepatitis $B$ core antigen ( $\mathrm{HBcAG}$ ) and hepatitis B e antigen ( $\mathrm{HBeAg}$ ) (Fig. 2). The respective antibodies of these antigens have been designated anti-HBs, anti-HBc, and anti-HBe. 
In the early 1970 s, the development of highly sensitive and specific tests to detect such hepatitis B markers as $\mathrm{HBsAg}$, anti-HBs, and anti-HBc provided the technology required to evaluate safety, immunogenicity, and efficacy of hepatitis B vaccine. Studies on the natural history of hepatitis B had revealed that induction of anti-HBs and/or anti-HBc was indicative of immunity.

Another milestone was reached in 1971 when my colleagues and I demonstrated that heat-inactivated MS-2 hepatitis serum was no longer infectious but remained antigenic (Krugman et al. 1971). Thus, the heat that inactivated HBV did not affect the antigenic potential of HBsAg. The detection of anti-HBs in the serum of recipients of this heat-inactivated preparation indicated that the noninfectious HBsAg particle was the antigen needed for vaccine production. Subsequent studies revealed that the heat-inactivated serum was not only immunogenic but also partially protective.

The results of our studies with heat-inactivated MS-2 hepatitis serum demonstrated that hepatitis B vaccine development was an achievable objective. These findings stimulated Hilleman and his colleagues and other investigators to accelerate their efforts to extract and purify antigen from HBsAg-positive plasma for subsequent vaccine development.

Vaccine development gathered momentum in 1973 when HBV infection was transmitted successfully to nonhuman primates (Barker et al. 1973). Seronegative chimpanzees proved to be excellent animal models for the study of hepatitis B infection and the evaluation of vaccines for safety and efficacy.

A third milestone was the development of safe, practical, and effective vaccines for routine application in man. Starting in 1968, Hilleman et al. (1975) developed a highly purified hepatitis B surface antigen vaccine that was first tested clinically in 1975 and proved highly effective not only in stimulating antibody but also in conferring protective immunity. Procedures for development of other hepatitis B vaccines have been described by Purcell and Gerin (1975), by Maupas et al. (1976), and by Reesink et al. (1978). The following discussion of the manufacturing process for the hepatitis B vaccine will summarize the procedures employed by Hilleman et al. to yield a highly purified product which, by incorporating various measures, assures safety from possible adventitious agents. The objective underlying the steps used in the process is to extract and purify plasma-derived HBsAg by utilizing biophysical technology capable of eliminating all possible living agents that may be present in human blood.

\section{Manufacturing Process}

Plasma obtained from hepatitis B carriers contains enormous quantities of $\mathrm{HBsAg}$ and small quantities of $\mathrm{HBV}$. The ratio may be as high as $10,000 \mathrm{HBsAg}$ particles to one HBV particle. The manufacturing process that was developed by Hilleman et al. (1975) involves a series of complex physical and chemical procedures to isolate the 20 -nm spherical, noninfectious particles. The procedures include concentration of $\mathrm{HBsAg}$ by ammonium sulfate precipitation, isopyknic banding in sodium bromide, and rate zonal centrifugation in sucrose. The partially purified $\mathrm{HBsAg}$ particles are then treated with pepsin at $\mathrm{pH} 2$, followed by 
treatment with $8 M$ urea to remove extraneous blood and human liver proteins. After gel filtration, the antigen is treated with formalin in a $1: 4,000$ dilution. The final step is formulation of the vaccine to contain $20 \mu \mathrm{g}$ of HBsAg and $0.5 \mathrm{mg}$ of $\mathrm{Al}^{3-}$ as aluminum hydroxide in each $1.0-\mathrm{ml}$ dose. Thiomersal, in a concentration of $1: 20,000$, is added as a preservative.

Each of these three steps, (pepsin, urea, and formalin) has been shown to inactivate $\mathrm{HBV}$ in chimpanzee studies. In addition, pepsin has been shown to inactivate viruses from every known group, including rhabdoviruses (vesicular stomatitis virus), poxviruses (vaccinia), togavirus (sindbis), herpesviruses (herpes simplex, type 1), coronaviruses (infectious bronchitis virus), and reovirus. The urea treatment inactivates myxoviruses (Newcastle disease virus), picornaviruses (mengovirus), and slow viruses (scrapie agent), as well as the viruses inactivated by pepsin. Formalin inactivates a wide variety of viruses, including non-A, non-B hepatitis viruses, parvoviruses, retroviruses, and the delta agent. However, formalin does not inactivate such viruses as SV40 virus, and inactivation can be inhibited by the presence of impurities. Therefore, the use of multiple purification and inactivation procedures provides assurance of inactivation of any agent that might be carried over from human blood, including the putative AIDS agent.

The putative AIDS agent was recently described in the U.S.A. (Gallo et al. 1984) and in France (Vilmer et al. 1984). This agent, though still unproved as the cause of AIDS, seems a likely candidate. It is of some interest that this agent is a retrovirus of the human T cell leukemia group of viruses (HTLV) and that representatives of the group are known to be inactivated by formaldehyde. Additionally, the surface glycoproteins of retroviruses are at least partially destroyed by proteolytic enzymes. This gives added evidential support for confidence that the process serves to destroy all known life forms.

Electronmicrographs of the original plasma and the purified HBsAg are shown in Fig. 3. All of the protein in the final product is accounted for as HBsAg,

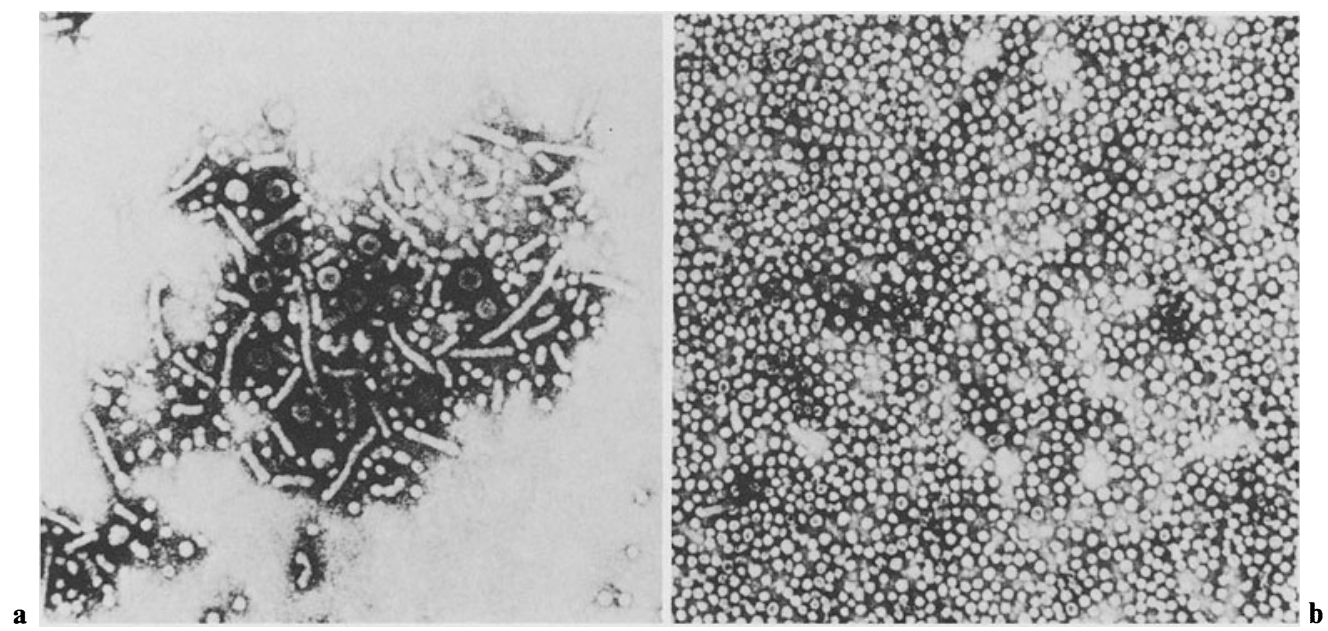

Fig. 3 a, b. Electronmicrographs a of plasma containing infectious $\mathrm{HBV}$ particles and excess $\mathrm{HBs} \mathrm{Ag}$ particles and $\mathbf{b}$ of hepatitis B vaccine (purified HBsAg particles) 
thereby decreasing the potential risk of an autoimmune response to extraneous protein.

Tests for safety of the vaccine include in vitro and in vivo assays for viral and microbial sterility of the bulk plasma, the purified antigen, and the final product. Each lot of vaccine is tested in chimpanzees to detect possible residual infectivity to hepatitis $B$ virus or other viral agents.

The initial studies in chimpanzees confirmed the safety, immunogenicity, and efficacy of the vaccine for these animals. Vaccine-induced anti-HBs protected immunized chimpanzees who were subsequently challenged with live $\mathrm{HBV}$. In contrast, unimmunized, seronegative chimpanzees contracted hepatitis B infection after a comparable challenge.

\section{Evaluation of the Hepatitis B Vaccine}

\section{Safety - Evaluation Before Licensure}

The first human trials with the vaccine prepared by Hilleman et al. were begun in November 1975. These initial studies involved 11 seronegative adult volunteers who received one dose of vaccine. Follow-up observation for 6 months revealed no evidence of HBV infection. Serial samples of blood at 1-month intervals revealed no detectable $\mathrm{HBsAg}$ or anti-HBc, and the serum aminotransferase (ALT) values were normal. Subsequent studies by various investigators from 1976 to 1978 confirmed the safety of the vaccine.

\section{Immunogenicity}

The inactivated hepatitis $\mathrm{B}$ vaccine has proved to be highly immunogenic when given intramuscularly in a three-dose schedule at 0,1 , and 6 months. The present recommended dose ist $10 \mu \mathrm{g}(0.5 \mathrm{ml})$ for children under 10 years of age and $20 \mu \mathrm{g}$ $(1.0 \mathrm{ml})$ for older children and adults. Immunocompromised persons, such as hemodialysis patients, should receive a $40-\mu \mathrm{g}$ dose $(2.0 \mathrm{ml})$ because they have not responded as well as healthy adults. After three $40-\mu \mathrm{g}$ doses of vaccine, the antiHBs response may range between $60 \%$ and $70 \%$. Hepatitis $\mathrm{B}$ vaccine will induce an anti-HBs response in $80 \%-90 \%$ of immunocompetent recipients by 1 month after the second dose. The third (booster) dose, given at 6 months, will increase the antibody response to over $95 \%$, as well as increasing the anti-HBs titers of those who responded previously. The immune response may be sluggish in individuals older than 40 years, and males do not respond as well as females. To date, vaccine-induced anti-HBs has persisted for at least 4 years in about $90 \%$ of vaccinees. Since antibody titers would be expected to decline, it is likely that an additional booster dose may be required 5 or more years after the third dose, unless it can be shown that exposure to HBV will induce an anamnestic response that will afford protection against this "long incubation disease."

In one reported study by Francis et al. (1982) involving 1,402 homosexual men, the antibody response after three doses of vaccine was $85 \%$ instead of $95 \%$. Subsequent investigation revealed that many vials of vaccine used in this study 
had been inadvertently frozen. It has been shown that freezing decreases the potency. The optimum temperature for shipment and storage of inactivated hepatitis $\mathrm{B}$ vaccine is $2^{\circ}-8^{\circ} \mathrm{C}$. It should not be frozen.

\section{Efficacy}

The efficacy of hepatitis B vaccine has been evaluated in three randomized, placebo-controlled, double-blind trials among 1,083 homosexual men in New York (Szmuness et al. 1980), 1,402 homosexual men in five additional cities (Francis et al. 1982), and 865 staff members of 43 hemodialysis units in the United States (Szmuness et al. 1982). The vaccine proved to be highly effective in preventing HBV infection and disease. The difference in cumulative attack rates between the vaccine and placebo groups was highly significant. The vaccine recipients were protected against acute hepatitis B, asymptomatic infection, and chronic HBsAg carrier state. All vaccine recipients who had an anti-HBs response were protected against disease. The only cases of hepatitis B disease in the vaccine group occurred in those who did not respond and in those who were already infected at the time of entry in the study. However, the vaccine appeared to be partially effective when given after exposure. In the hemodialysis study, the vaccine which contained HBsAg subtype ad protected medical staff exposed to patients suffering from HBsAg subtype ay infection.

\section{Safety - Evaluation Since Licensure}

Observation of the 3,350 persons who participated in the double-blind, placebocontrolled trials revealed that the only increased side effect in those who received vaccine was transient, local soreness at the site of inoculation. The frequency of fever and other complaints was the same in placebo and vaccine groups, and no serious vaccine-related reactions were observed. In September 1982, a Public Health Service Inter-Agency Group reported that 19,000 persons were immunized in studies prior to licensure between October 1975 and December 1981 (United States Public Health Service Center for Disease Control 1982). No serious side effects were reported among members of this group.

A subsequent report in March (MMWR 1983) reviewed the reactions of about 200,000 individuals who received the vaccine after its licensure in 1981. Based on previous double-blind, placebo-controlled studies, the reported side effects and illnesses that occurred within 6 weeks of immunization did not appear to be causally related to hepatitis $B$ vaccine. The various manifestations reported did not exceed the number of such events that would be expected to have occurred by chance alone within 6 weeks of vaccinating 200,000 people.

As of June 1984, more than 500,000 persons in the United States and an additional 500,000 persons worldwide have been immunized with the plasma-derived hepatitis $B$ vaccine. To date, no serious side effects have been attributed to the use of vaccine. For example, the speculation and concern about a possible association between acquired immune deficiency disease (AIDS) and hepatitis B vaccine have proved to be groundless. The lack of association is based on three 
findings: (a) no cases of AIDS have been detected among the many thousands of health professionals who have been immunized during the past 2 years; (b) no increased incidence of AIDS has been observed among immunized high-risk male homosexuals when compared with unimmunized high-risk homosexuals, and (c) it would be incredible that the putative AIDS agent could survive the inactivating procedures involving pepsin at $\mathrm{pH} 2,8 \mathrm{M}$ urea, and formalin. The physical and biochemical procedures involved in the preparation of hepatitis B vaccine are capable of eliminating all of the agents proposed as possible causes of AIDS.

\section{Conclusion}

The first-generation, plasma-derived hepatitis B vaccines have proved to be safe, immunogenic, and effective. These licensed products are recommended for persons of all ages who are at high risk of contracting hepatitis B infection. Studies currently in progress indicate that future second-generation vaccines will be prepared by DNA recombinant technology. In the meantime, extensive experience during the past 2 years has revealed that the demonstrated benefits of the currently licensed plasma-derived vaccines far exceed any theoretical risks.

\section{References}

Barker LF, Chisari FV, McGrath PP, et al. (1973) Transmission of type B viral hepatitis to chimpanzees. J Infect Dis 127:648-662

Blumberg BS, Alter HJ, Visnich S (1965) A “new" antigen in leukemia sera. JAMA 191:541-546

Dane DS, Cameron CH, Briggs M (1970) Virus-like particles in serum of patients with Australia antigen-associated hepatitis. Lancet 1:695-698

Francis DP, Hadler SC, Thompson SE, et al. (1982) The prevention of hepatitis B with vaccine. Ann Intern Med 97:362-366

Gallo RC, Salahuddin SZ, Popovic M, et al. (1984) Frequent detection and isolation of cytopathic retroviruses (HTLV-III) from patients with AIDS and at risk for AIDS. Science 224:550-552

Hilleman MR, Buynak EB, Roehm RR, et al. (1975) Purified and inactivated human hepatitis B vaccine: progress report. Am J Med Sci 270:401-403

Krugman S, Giles JP, Hammond J (1971) Viral hepatitis, type B (Ms-2 strain): studies on active immunization. JAMA 217:41-45

Maupas P, Goudeau A, Coursaget P, et al. (1976) Immunization against hepatitis B in man. Lancet 1:1367-1370

Prince AM (1968) An antigen detected in the blood during the incubation period of serum hepatitis. Proc Natl Acad Sci USA 60:814-821

Purcell RH, Gerin JL (1975) Hepatitis B subunit vaccine: a preliminary report of safety and efficacy tests in chimpansees. Am J Med Sci 270:395-399

Reesink HW, Brummelhuis EH, van Elven LJT, et al. (1978) The preparation and evaluation of a hepatitis B vaccine in the Netherlands. In: Vyas GN, Cohen SN, Schmidt R (eds) Franklin Institute, Philadelphia, p 714

Szmuness W, Stevens CE, Harley EJ, et al. (1980) Hepatitis B vaccine: demonstration of efficacy in a controlled clinical trial in a high risk population in the United States. N Engl J Med 303:833-841

United States Public Health Service Centers for Disease Control (1982) Hepatitis B vaccine safety: report of an inter-agency group. MMWR 31:465-467

United States Public Health Service Centers for Disease Control (1983) The safety of hepatitis B vaccine. MMWR 32:134-136

Vilmer E, Barre-Sinoussi F, Rouzioux C, et al. (1984) Isolation of new lymphotropic retrovirus from two siblings with hemophilia B, one with AIDS. Lancet 1:753-757 